\title{
Spatially Resolved Cathodoluminescence in the Vicinity of Defects in the High-Efficiency InGaN/GaN Blue Light Emitting Diodes
}

\author{
Manh-Ha Doan ${ }^{1,2}$ and Jaejin Lee ${ }^{1}$ \\ ${ }^{1}$ Department of Electrical and Computer Engineering, Ajou University, Suwon 443-749, Republic of Korea \\ ${ }^{2}$ Department of Energy Science, Sungkyunkwan University, Suwon 440-746, Republic of Korea \\ Correspondence should be addressed to Jaejin Lee; jaejin@ajou.ac.kr
}

Received 24 May 2014; Revised 14 August 2014; Accepted 20 August 2014; Published 1 September 2014

Academic Editor: Mohindar S. Seehra

Copyright (C) 2014 M.-H. Doan and J. Lee. This is an open access article distributed under the Creative Commons Attribution License, which permits unrestricted use, distribution, and reproduction in any medium, provided the original work is properly cited.

\begin{abstract}
In addition to the standard $447 \mathrm{~nm}$ blue emission from the InGaN/GaN multiple quantum wells, a high-energy shoulder is clearly observed in cathodoluminescence spectra of the high-efficiency InGaN/GaN blue light emitting diodes grown on sapphire substrates by metalorganic chemical vapor deposition. Monochromatic cathodoluminescence images of the samples measured at low temperature reveal a competition between the two emissions in the vicinity of the dislocations. The high-energy emission is dominant at the regions near the dislocation cores, while the blue emission is enhanced around the dislocation edges. The highenergy emission region is considered as a potential barrier that prevents the carriers for the blue emission from nonradiatively recombining at the dislocations.
\end{abstract}

\section{Introduction}

InGaN compounds have emerged as important semiconductor materials with applications for optoelectronic devices such as light emitting diodes (LEDs), laser diodes, and photovoltaics [1]. Despite the high dislocation density, blue GaNbased LEDs grown on sapphire substrates using InGaN/GaN multiple quantum well (MQW) active layers usually show a high internal quantum efficiency $[2,3]$. Although there have been numerous proposals to explain this phenomenon, it still seems to be a controversial issue. The inhomogeneity of In composition and/or quantum well width in the InGaN/GaN MQW active layer that induces a potential fluctuation for carrier localization is usually considered to be one of the main origins of its high-emission efficiency [3-10]. It is believed that a formation of In-rich quantum dots observed in the MQW regions induces the localized energy states that make the carriers recombine radiatively before they are capped by nonradiative recombination centers at the dislocations $[4,5]$. However, there have been several experimental observations on the unwanted In-rich clusters in the InGaN/GaN
MQW regions that can be formed by irradiation of a highenergy electron beam during the investigation of the MQW structures using a transmission electron microscopy $[6,7]$. Even though some authors recently report that In clustering is not present in their as-grown InGaN QWs through the use of aberration-corrected electron microscopy below the knock-on threshold [8], it is still difficult to conclude that large-scale compositional fluctuations do not occur in any InGaN/GaN MQW structure. In addition to the gross well width fluctuation observed in the entire QW region [9], it is also considered that the formation of $\mathrm{V}$-shaped pits at the threading dislocations induces the side-wall quantum wells with the reduced thickness and higher band gap energy [10]. These thinner wells can act as potential barriers around the defects, which keep carriers from nonradiatively recombining. Other reports, however, show that the $\mathrm{V}$-shaped pits are filled in by a p-GaN during the growth of the top cladding layer at high temperature [11], and side-wall quantum wells may be just interference fringes arising from lattice strain [12].

Cathodoluminescence (CL) measurement is well known to be one of the powerful techniques for the investigation 
of recombination processes in semiconductors. In addition, from its spatially resolved luminescence profile, one can estimate a dislocation distribution inside the sample without destructing its structure. There have been numerous researches on using the $\mathrm{CL}$ measurements to examine the properties of the GaN as well as InGaN/GaN MQWs $[13,14]$. However, to the best of our knowledge, there have not been any reports on CL mapping of a high-efficiency InGaN/GaN blue LED structure with an additional high-energy emission. In this study, we (i) employ the CL measurement technique to investigate the optical and structural properties of the InGaN/GaN blue LED and (ii) correlate luminescence mapping results with the high-resolution transmission electron microscopy (HRTEM) and atomic force microscopy (AFM) observations to study the possible origin of its high quantum efficiency.

\section{Experimental}

The blue InGaN/GaN LED structure with an expected emission wavelength around $440 \mathrm{~nm}$ is grown by low-pressure metalorganic chemical vapor deposition (LP-MOCVD) on patterned sapphire substrates (PSS). It is well known that using a PSS for the growth of GaN-based LEDs can significantly improve the device performances $[15,16]$. Prior to the growth of the LED structure, the hexagonal arrays of the hemispherical patterns with a diameter of $3 \mu \mathrm{m}$ are fabricated on the c-plane sapphire substrate using an ICP etching method. The detailed processes can be found elsewhere [16]. The LED structure includes a $30 \mathrm{~nm}$ lowtemperature GaN nucleation layer, $1 \mu \mathrm{m}$ undoped GaN, $4 \mu \mathrm{m}$ Si-doped n-GaN, six $\operatorname{In}_{0.3} \mathrm{Ga}_{0.7} \mathrm{~N} / \mathrm{GaN}$ MQWs, $20 \mathrm{~nm} \mathrm{p}-$ AlGaN electron-blocking layer, and an $\mathrm{Mg}$-doped $\mathrm{p}-\mathrm{GaN}$ top cladding layer of $0.25 \mu \mathrm{m}$. Crystal quality and structural properties of the LEDs are examined by the high-resolution X-ray diffraction, HRTEM, and AFM. It is noted that our LED devices which are fabricated using the above InGaN/GaN MQW structure show an external quantum efficiency of up to $24.2 \%$ (light output power of $13.31 \mathrm{~mW}$ at $20 \mathrm{~mA}$ current injection). Taking into account a light extraction efficiency of about $40 \%$ for a similar LED structure as calculated [17], an internal quantum efficiency of about $60 \%$ could be expected from our InGaN/GaN MQW structure. CL measurements are carried out to investigate the optical properties of the InGaN/GaN LEDs. The CL measurements are performed using a $10 \mathrm{kV}$ electron beam with a Gatan MonoCL3+ system equipped with a high-sensitivity photomultiplier tube (PMT) (thermoelectrically cooled to $-20^{\circ} \mathrm{C}$ ) attached to a scanning electron microscopy (SEM) (S-4300SE from Hitachi). For TEM observations, bright-field TEM images and HRTEM micrographs are collected by using a JEOL JEM $2100 \mathrm{~F}$ system operating at $200 \mathrm{kV}$.

\section{Results and Discussion}

Figure 1 shows a CL spectrum of the InGaN/GaN LEDs measured at room temperature. A high-intensity emission peaked at around $447 \mathrm{~nm}$ is from the InGaN/GaN MQW

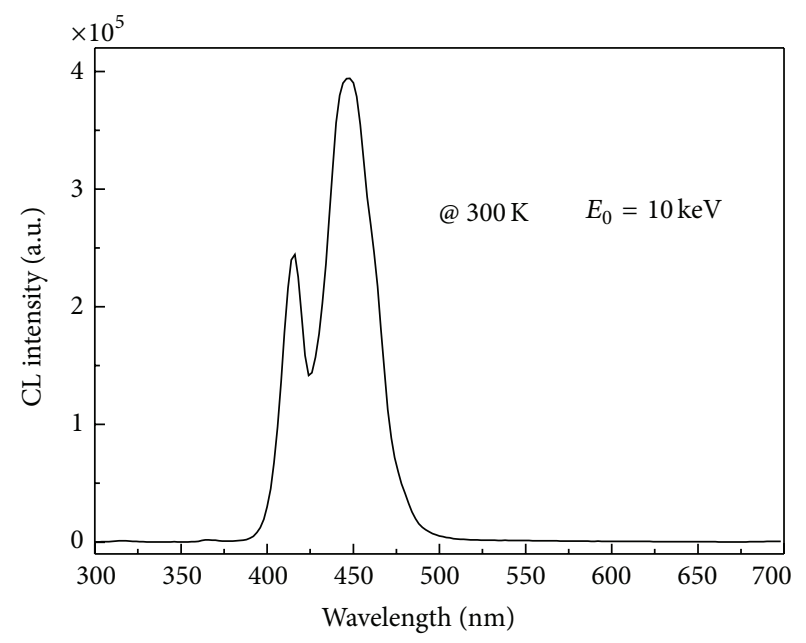

FIGURE 1: Room-temperature CL spectrum of the InGaN/GaN blue LED structure.

active layer. GaN band-edge emission peak around $365 \mathrm{~nm}$ is difficult to be observed because it is too weak compared to that from the MQW. It is interesting to note that a clearly distinguishable high-energy emission shoulder which peaked at around $415 \mathrm{~nm}$ is observed in the CL spectrum of our InGaN/GaN LEDs. There have been a lot of reports on the additional higher-energy emission from the highefficiency InGaN/GaN MQW LED structures [10, 18, 19]. The origin of this emission peak, however, seems to be still unclear. O'Donnell et al. considered that the highly restricted solubility of In in $\mathrm{GaN}$ (less than 6\% at typical temperature) leads to an InGaN active layer composed of quantum dots of nearly uniform composition (approaching InN) embedded in an In deficient matrix $\left(\operatorname{In}_{x} \mathrm{Ga}_{1-x} \mathrm{~N}\right.$, with $\left.x<0.06\right)$ [18]. Some authors believe that the high-energy emission comes from the InGaN/GaN MQWs with thinner well widths at the $\mathrm{V}$-pit defect positions [10]. On the other hand, other groups concluded that this emission is from the stacking faults at the well/barrier interfaces in the MQW region [19].

Figure 2 shows a cross-section HRTEM image of the InGaN/GaN MQW region in the studied samples. The inset shows a large magnification of the 6 quantum wells. We observed smooth interfaces at the InGaN/GaN MQWs with their thickness and periodicity of approximately 2.5 and $6.5 \mathrm{~nm}$, respectively, except at the positions of the threading dislocations (TD). The InGaN/GaN quantum well structures are not well-defined around TD area, which is similar to the observation of Sharma et al. [11]. Within the resolution limit of the HRTEM measurement, we have not observed any Vside wall InGaN/GaN quantum wells or stacking faults at the well/barrier interfaces. Figure 3 shows an AFM image of the InGaN/GaN LED surface. The V-pit defects with an average depth of $2.5 \mathrm{~nm}$ and density of about $9 \times 10^{7} \mathrm{~cm}^{-2}$ are observed on the surface of the samples. It is noted that this $\mathrm{V}$-pit density is slightly smaller compared to the TD density estimated by using cross-sectional TEM as reported in [15] as well as our CL mapping observations shown below. It may be attributed to the fact that some $\mathrm{V}$-shaped defects formed at 


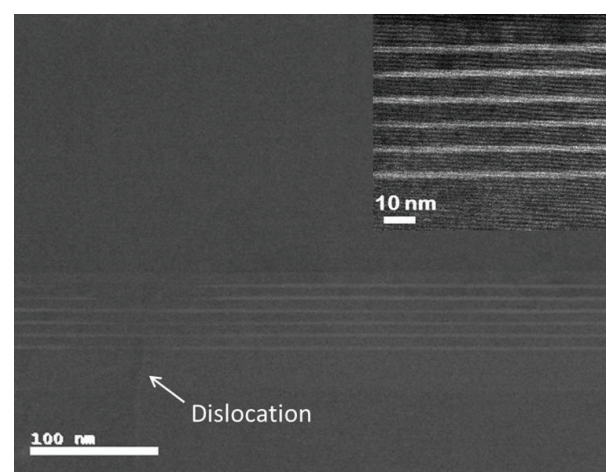

FIGURE 2: Cross-sectional HRTEM image of the InGaN/GaN MQW region in the LED. The bright rows are the InGaN layers. Some MQWs are not able to be observed at the position of the TD. The inset shows a large magnification of the MQWs; there are not any side-wall wells, stacking faults at the InGaN/GaN interfaces, or socalled InGaN quantum dots observed in the MQWs region.

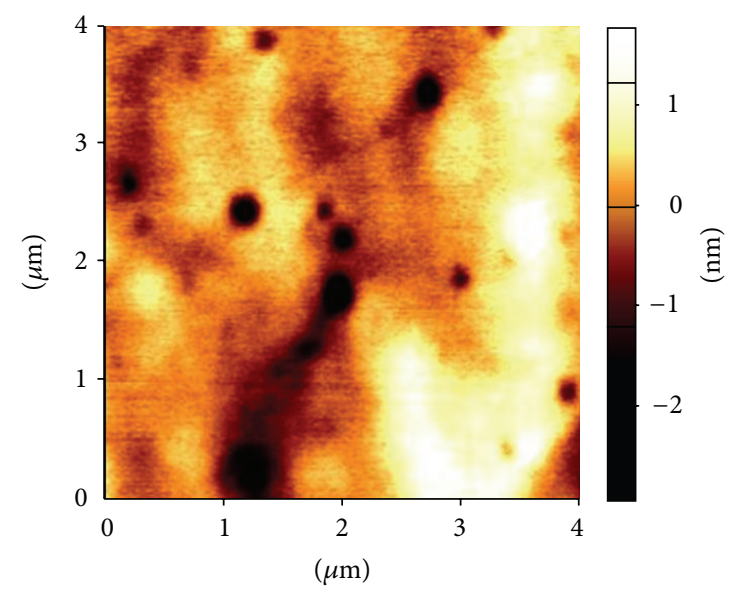

FIGURE 3: AFM image of the InGaN/GaN LED surface. The V-pits with average depth of $2.5 \mathrm{~nm}$ and density of about $9 \times 10^{7} \mathrm{~cm}^{-2}$ are observed on the surface of the sample.

the TD positions inside the LED structure had been filled in by a $\mathrm{p}-\mathrm{GaN}$ as discussed in [11]. As a result, they become so shallow and cannot be detected by the AFM measurements even when we used a high-resolution scanning for a small area. Our HRTEM and AFM observations suggest that the origin of the high-energy emission in Figure 1 may be from other mechanisms but not from the V-side wall wells, the defects at the well/barrier interfaces, or the InGaN quantum dots as introduced in $[10,18,19]$.

We employed a CL mapping technique to investigate the origin of the high-energy emission in our InGaN/GaN LED structures. There are two ways in obtaining the CL signal from the specimen. One is panchromatic CL images that are formed by plotting integrated luminescence for the wavelength range $(160-930 \mathrm{~nm})$ of the PMT detector at each point of the electron beam irradiation. The other is monochromatic CL (mono-CL) images that only luminescence at particular wavelength is plotted by adjusting the spectrometer. Figure 4 shows the mono-CL images of the sample at room temperature corresponding to the two emission peaks in Figure 1. It is difficult to find an obvious correlation between the high-energy [Figure 4(a)] and the blue [Figure 4(b)] emissions but we may conjecture that this is due to diffusion of electrons and generated-carriers at room temperature. To clarify the competitive relationship between the two emissions, we carried out the CL measurements at low temperatures by using a liquid $\mathrm{N}_{2}$ to decrease the sample chamber temperature down to $77 \mathrm{~K}$. From the lowtemperature CL spectra of the samples (not shown here), we also observed two strong emission peaks centered at around 418 and $446 \mathrm{~nm}$. Figure 5 shows the SEM and mono-CL images of the LEDs recorded at the wavelengths corresponding to the peak emissions of the GaN, high-energy, and blue emissions from the InGaN/GaN MQWs. Although the V-pit defects on the surface of the sample are not able to be seen in the SEM image [Figure 5(a)], dislocations inside the LED structure can be observed in the CL images as in Figures 5(b)-5(d). It is well known that the TDs in the GaN films usually act as nonradiative recombination centers and leave the dark regions in the CL images $[13,14]$. The density of the TD in the sample is estimated to be about $1.5 \times 10^{8} \mathrm{~cm}^{-2}$ from the number of dark spots in Figure 5(b). This value is well consistent with the dislocation density in the LED grown on the PSS observed by the TEM investigations [15]. There are competitions between the two, high-energy and blue, emissions as observed in the CL images of the sample [Figures 5(c) and 5(d)]. The high-energy emission is dominant at the positions near the center of the dislocation [Figure 5(c)] while these positions show dark spots in the CL image recorded at the blue emission peak [Figure 5(d)]. Intensity of the main blue emission is enhanced at regions which surround the high-energy emission areas as in Figure 5(d). From our CL and TEM observations, we believe that this emission is from the vicinity of the dislocations in the LED structures. This may be attributed to the reduction of the quantum well thickness and/or the In composition near the dislocation positions. In fact, it is reported that there is an accumulation of In in the wells close to the dislocations, followed by depletion just outside the dislocation core and spike at the dislocations. Sometimes the In depletion outside the core is so severe that no wells can be seen in the TEM images [20]. It has been noted that when we use a laser lift-off (LLO) technique to remove the sapphire substrate and transfer LED structure to a metal carrier for the fabrication of a vertical LED structure, the high-energy emission from the vertical LED is dominant (compared to the blue emission) and its peak slightly shifts to a shorter wavelength. It is reported that defects and dislocations can be generated in the LED structure during the LLO process [21]. We thus consider that the high-energy emission may be mainly from the In depletion at the dislocation position. Then after the LLO process, the In diffusion is enhanced resulting in a dominance of the high-energy emission and its blue shift.

From above results and discussion, it is suggested that the high-energy area near the dislocation core acts as a potential barrier that prevents the carriers with smaller energy (for blue emission) from moving to the dislocations. The potential barrier is calculated to be about $230 \mathrm{meV}$ from the CL spectra 


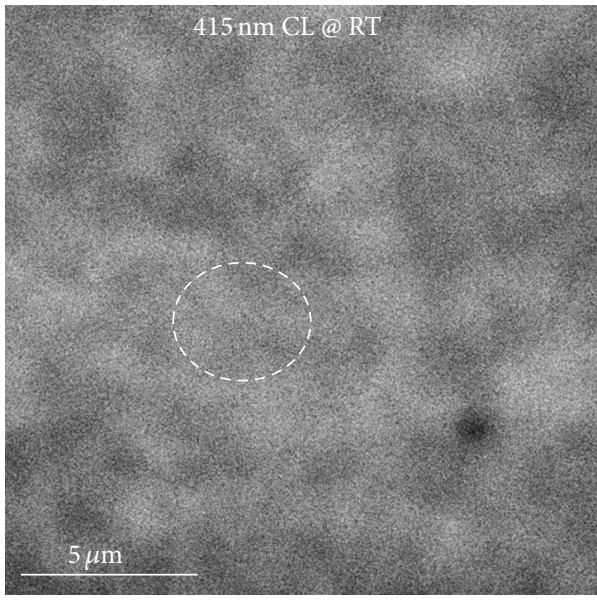

(a)

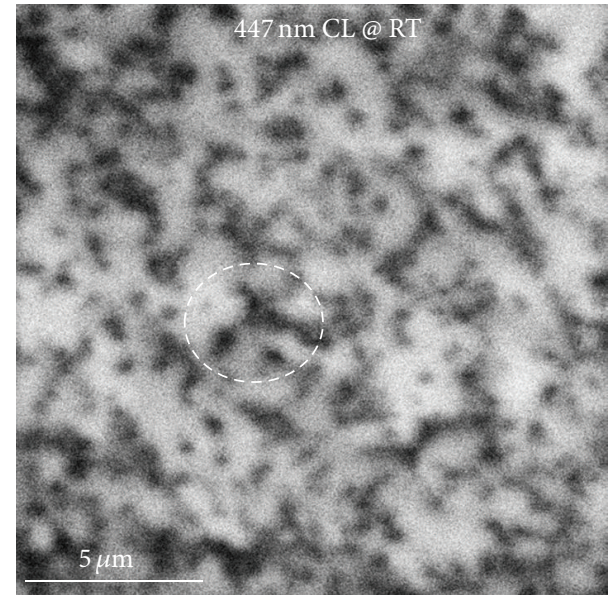

(b)

Figure 4: Observed mono-CL images which correspond to the high-energy and blue emission at room temperature. The dash circles show regions with a competition of the two emissions. The competition is not obvious at room temperature.

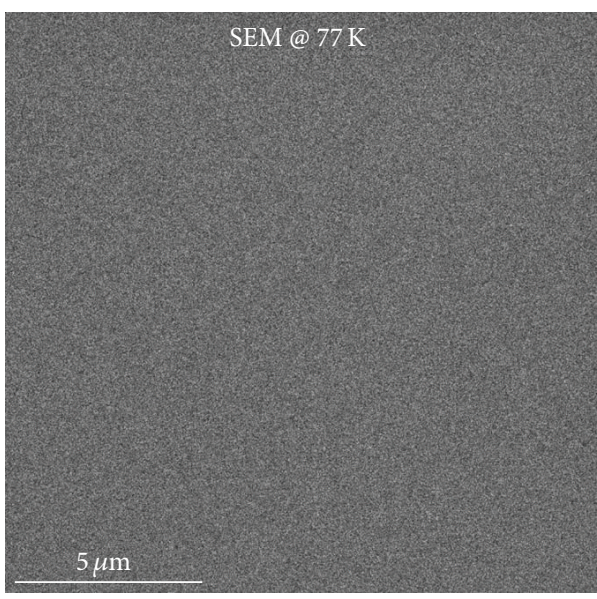

(a)

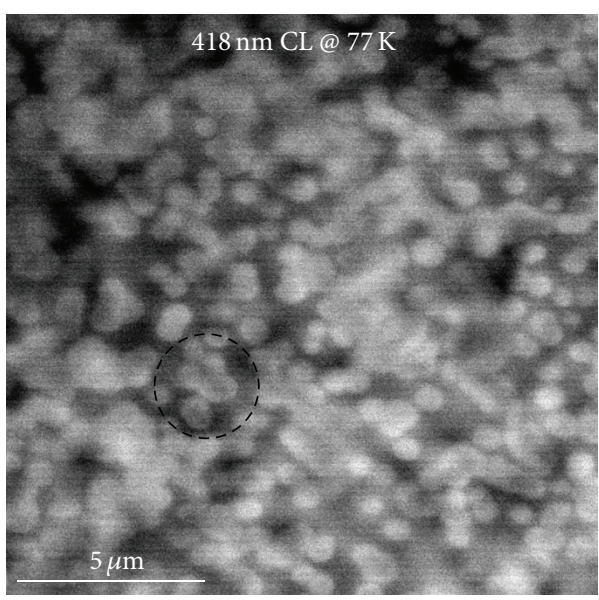

(c)

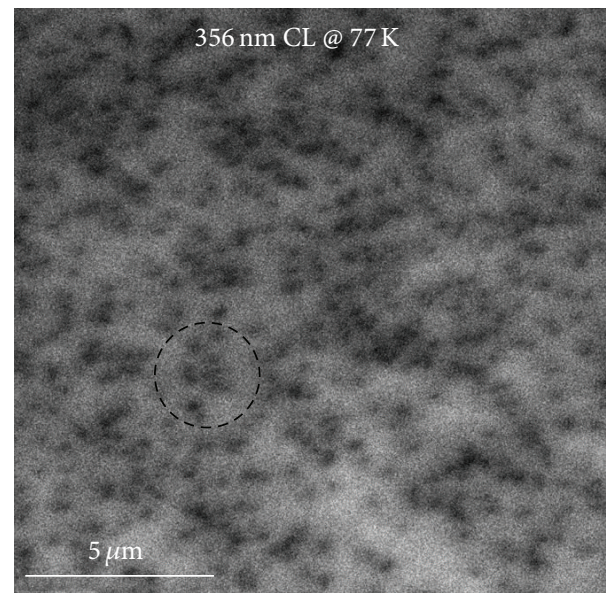

(b)

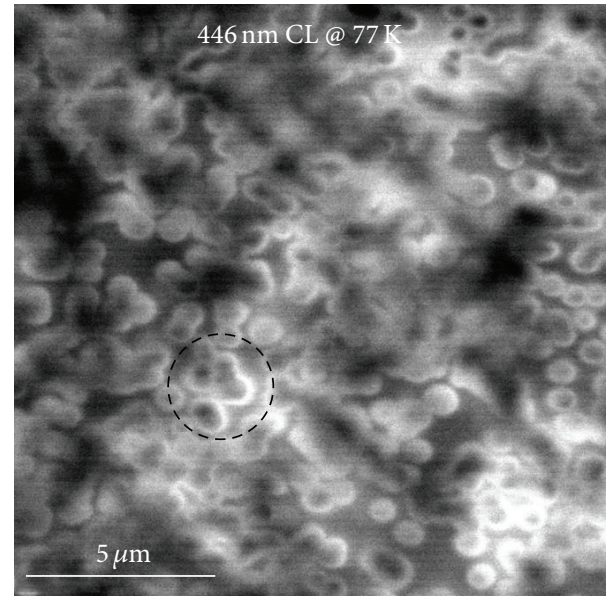

(d)

FIgure 5: (a) SEM and ((b)-(d)) mono-CL images of the InGaN/GaN LEDs at low temperature. The CL images in ((b)-(d)) correspond to GaN, high-energy, and blue emission, respectively. The V-pits on the surface are not observed by the SEM measurements because of their shallow depth. Dark spots in (b) correspond to the dislocations in the LED structure. The dashed circles in (b)-(d) show competitions of the emissions around the dislocations. 
of the LEDs, which is much higher than the thermal energy of the carriers at room temperature. As a result, the carriers related with the main blue emission may not pass through the potential barrier and recombine radiatively before arriving at the dislocations.

\section{Conclusions}

In summary, we used HRTEM, AFM, and CL mapping measurements to investigate the origin of the high-energy emission in the high-efficiency InGaN/GaN blue LEDs. The $\mathrm{CL}$ images of the sample at low temperature reveal that the high-energy emission is dominant in the vicinity of the dislocation core. It may be attributed to the reduction of In composition in the InGaN/GaN MQWs at regions near the dislocations. The high-energy area acts as the potential barriers that keep the carriers for the blue emission from being captured by the dislocations. Our proposed mechanism may support for the observed high internal quantum efficiency in the InGaN/GaN blue LED structure with a high dislocation density.

\section{Conflict of Interests}

The authors declare that there is no conflict of interests regarding the publication of this paper.

\section{Acknowledgment}

This work is supported by the National Research Foundation Grants (Mid-career Researcher Program: 2009-0083803).

\section{References}

[1] J. Wu, "When group-III nitrides go infrared: new properties and perspectives," Journal of Applied Physics, vol. 106, no. 1, Article ID 011101, 2009.

[2] S. D. Lester, F. A. Ponce, M. G. Crawford, and D. A. Steigerwald, "High dislocation densities in high efficiency GaN-based light-emitting diodes," Applied Physics Letters, vol. 66, no. 10, pp. 1249-1251, 1995.

[3] S. Nakamura, "The roles of structural imperfections in InGaNbased blue light-emitting diodes and laser diodes," Science, vol. 281, no. 5379, pp. 956-961, 1998.

[4] S. F. Chichibu, A. Uedono, T. Onuma et al., "Origin of defectinsensitive emission probability in In-containing (Al,In,Ga)N alloy semiconductors," Nature Materials, vol. 5, no. 10, pp. 810816, 2006.

[5] Y. Narukawa, Y. Kawakami, M. Funato, S. Fujita, and S. Nakamura, "Role of self-formed InGaN quantum dots for exciton localization in the purple laser diode emitting at $42 \mathrm{~nm}$," Applied Physics Letters, vol. 70, no. 8, pp. 981-983, 1997.

[6] J. P. O’Neill, I. M. Ross, A. G. Cullis, T. Wang, and P. J. Parbrook, "Electron-beam-induced segregation in InGaN/GaN multiplequantum wells," Applied Physics Letters, vol. 83, no. 10, pp. 19651967, 2003.

[7] T. Li, E. Hahn, D. Gerthsen et al., "Indium redistribution in an InGaN quantum well induced by electron-beam irradiation in a transmission electron microscope," Applied Physics Letters, vol. 86, no. 24, Article ID 241911, pp. 1-3, 2005.
[8] K. H. Baloch, A. C. Johnston-Peck, K. Kisslinger, E. A. Stach, and S. Gradečak, "Revisiting the "In-clustering" question in InGaN through the use of aberration-corrected electron microscopy below the knock-on threshold," Applied Physics Letters, vol. 102, no. 19, Article ID 191910, pp. 91910-91914, 2013.

[9] N. K. van der Laak, R. A. Oliver, M. J. Kappers, and C. J. Humphreys, "Role of gross well-width fluctuations in bright, green-emitting single InGaN/GaN quantum well structures," Applied Physics Letters, vol. 90, no. 12, Article ID 121911, 2007.

[10] A. Hangleiter, F. Hitzel, C. Netzel et al., "Suppression of nonradiative recombination by V-shaped pits in GaInN/GaN quantum wells produces a large increase in the light emission efficiency," Physical Review Letters, vol. 95, no. 12, Article ID 127402, pp. 127402-127404, 2005.

[11] N. Sharma, P. Thomas, D. Tricker, and C. Humphreys, "Chemical mapping and formation of V-defects in InGaN multiple quantum wells," Applied Physics Letters, vol. 77, no. 9, pp. 12741276,2000

[12] K. Ding and Y. Zeng, "Do sidewall quantum wells exist in GaInN-Based light-emitting diodes?" Japanese Journal of Applied Physics, vol. 49, no. 2, Article ID 028001, 2010.

[13] N. Ino and N. Yamamoto, "Low temperature diffusion length of excitons in gallium nitride measured by cathodoluminescence technique," Applied Physics Letters, vol. 93, no. 23, Article ID 232103, 2008.

[14] J. C. Brooksby, J. Mei, and F. A. Ponce, "Correlation of spectral luminescence with threading dislocations in green-lightemitting InGaN quantum wells," Applied Physics Letters, vol. 90, no. 23, Article ID 231901, 2007.

[15] J.-H. Lee, J.-T. Oh, J.-S. Park et al., "Improvement of luminous intensity of InGaN light emitting diodes grown on hemispherical patterned sapphire," Physica Status Solidi (C), vol. 3, pp. 2169-2173, 2006.

[16] J.-H. Lee, D.-Y. Lee, B.-W. Oh, and J.-H. Lee, "Comparison of InGaN-based LEDs grown on conventional sapphire and cone-shape-patterned sapphire substrate," IEEE Transactions on Electron Devices, vol. 57, no. 1, pp. 157-163, 2010.

[17] H. Kim, K.-K. Choi, K.-K. Kim et al., "Light-extraction enhancement of vertical-injection GaN-based light-emitting diodes fabricated with highly integrated surface textures," Optics Letters, vol. 33, no. 11, pp. 1273-1275, 2008.

[18] K. P. O'Donnell, R. W. Martin, and P. G. Middleton, "Origin of luminescence from InGaN diodes," Physical Review Letters, vol. 82, no. 1, pp. 237-240, 1999.

[19] C. L. Yang, L. Ding, J. N. Wang et al., "Thermally activated carrier transfer processes in InGaN/GaN multi-quantum-well light-emitting devices," Journal of Applied Physics, vol. 98, no. 2, Article ID 023703, 2005.

[20] N. Duxbury, U. Bangert, P. Dawson et al., "Indium segregation in InGaN quantum-well structures," Applied Physics Letters, vol. 76, no. 12, pp. 1600-1602, 2000.

[21] M. H. Doan, S. Kim, J. J. Lee, H. Lim, F. Rotermund, and K. Kim, "Influence of laser lift-off on optical and structural properties of In GaN/GaN vertical blue light emitting diodes," AIP Advances, vol. 2, no. 2, Article ID 022122, 2012. 

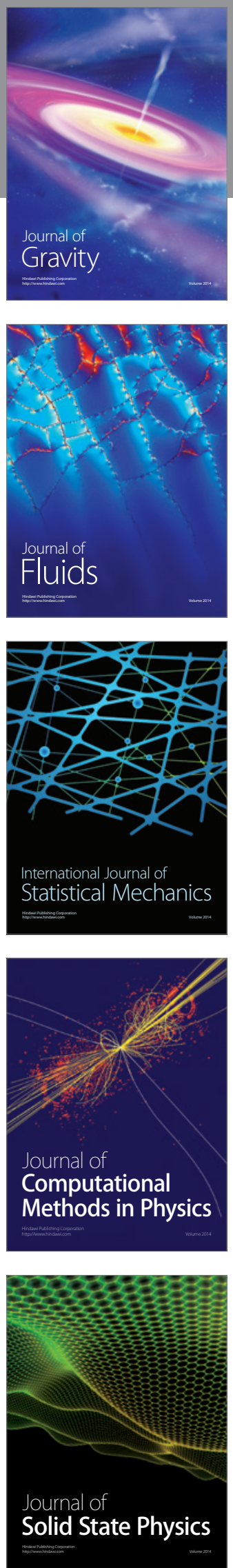

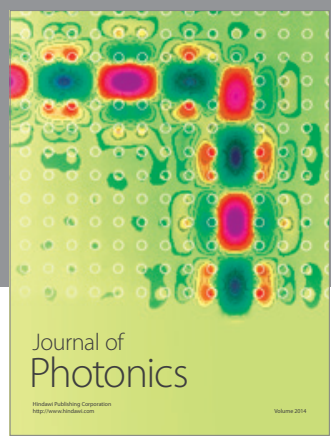

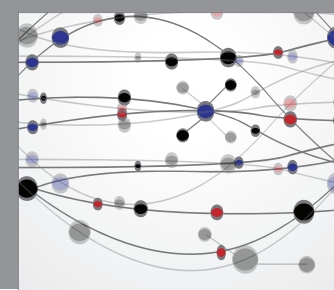

The Scientific World Journal

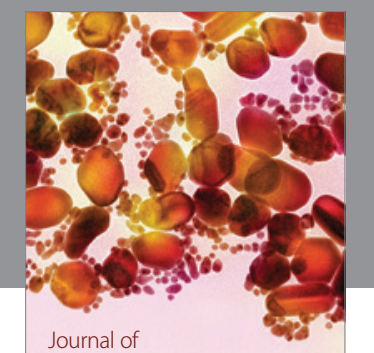

Soft Matter
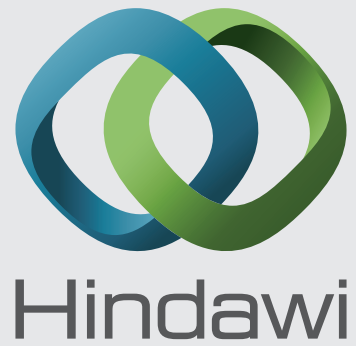

Submit your manuscripts at

http://www.hindawi.com
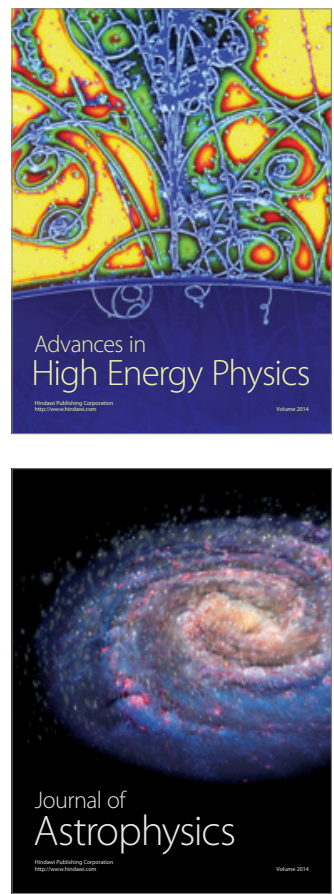
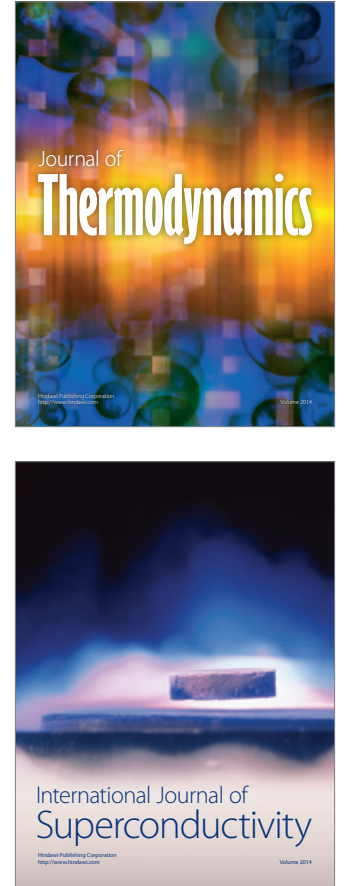
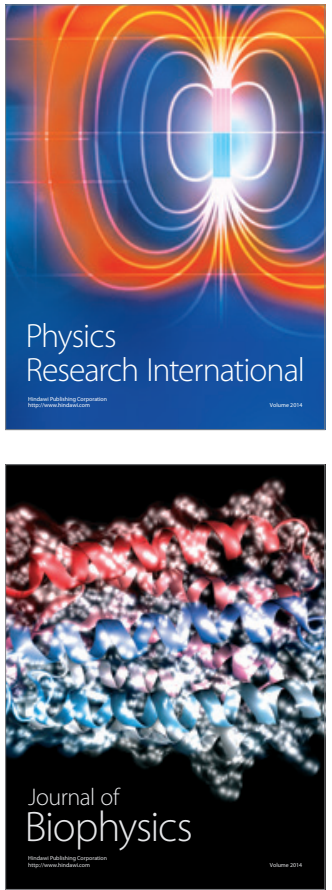
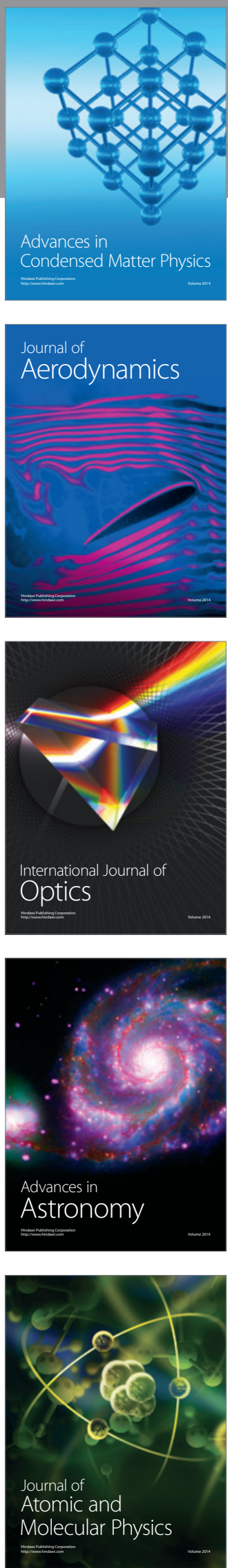